\title{
Young stars and reflection nebulae near the lower "edge" of the Galactic molecular disc ${ }^{\star}$
}

\author{
P. M. Palmeirim and J. L. Yun
}

\begin{abstract}
Universidade de Lisboa - Faculdade de Ciências, Centro de Astronomia e Astrofísica da Universidade de Lisboa, Observatório Astronómico de Lisboa, Tapada da Ajuda, 1349-018 Lisboa, Portugal e-mail: [ppalm;yun]@oal.ul.pt
\end{abstract}

Received 1 September 2009 / Accepted 16 November 2009

\begin{abstract}
Aims. We investigate the star formation occurring in a region well below the Galactic plane towards the optical reflection nebula ESO 368-8 (IRAS 07383-3325). We confirm the presence of a small young stellar cluster (or aggregate of tens of YSOs) identified earlier, embedded in a molecular cloud located near the lower "edge" of the Galactic disc, and characterise the young stellar population. We report the discovery of a near-infrared nebula, and present a $\mathrm{CO}$ map revealing a new dense, dynamic cloud core. Methods. We used near-infrared $J H K_{\mathrm{S}}$ images obtained with VLT/ISAAC, millimetre CO spectra obtained with the SEST telescope, and optical $V$-band images from the YALO telescope.

Results. This star formation region displays an optical reflection nebula (ESO 368-8) and a near-infrared nebula located about 46" $(1.1 \mathrm{pc})$ from each other. The two nebulae are likely to be coeval and to represent two manifestations of the same single star formation episode with about $1 \mathrm{Myr}$ age. The near-IR nebula reveals an embedded, optically and near-IR invisible source whose light scatters off a cavity carved by previous stellar jets or molecular outflows and into our line-of-sight. The molecular cloud is fully covered by our $\mathrm{CO}(J=1-0)$ maps and, traced by this line, extends over a region of $\sim 7.8 \times 7.8 \mathrm{pc}^{2}$, exhibiting an angular size $\sim 5.4^{\prime} \times 5.4^{\prime}$ and shape (close to circular) similar to spherical (or slightly cometary) globules. Towards the direction of the near-IR nebula, the molecular cloud contains a dense core where the molecular gas exhibits large line widths indicative of a very dynamical state, with stirred gas and supersonic motions. Our estimates of the mass of the molecular gas in this region range from 600 to $1600 M_{\odot}$. The extinction $A_{V}$ towards the positions of the optical reflection nebula and of the near-IR nebula was found to be $A_{V} \simeq 3-4$ mag and $A_{V} \simeq 12-15$ mag, respectively.
\end{abstract}

Key words. stars: formation - stars: pre-main sequence - ISM: clouds - infrared: stars - dust, extinction ISM: individual objects: ESO 368-8 (IRAS 07383-3325)

\section{Introduction}

Since early years, optical reflection nebulae have attracted much attention by their extended morphology and clearly nonpoint-like appearance (e.g. Hubble 1922; Barnard 1913, 1927; van den Bergh 1966). In the vicinity of reflection nebulae, different types of objects have been found, many associated with young stars and the process of star formation (e.g. emission-line stars, Herbig-Haro objects, jets and outflows, etc.). For a considerable period, these nebulae were among the best tracers of recent star formation ocurring in a region of the sky.

Subsequently, many infrared star clusters and stellar groups have been found during surveys towards directions of optical and radio HII regions. Testi et al. (1998) found sources around Herbig AeBe stars, part of them related to reflection nebulae. Soares \& Bica $(2002,2003)$ studied four low-mass star clusters in the CMaR1 molecular cloud, three of them related to optical reflection nebulae. Dutra et al. (2003) and Bica et al. (2003) investigated more than 3000 optical and radio nebulae with 2MASS (Skrutskie et al. 1997) and found 346 new embedded star clusters, stellar groups, and candidates.

Star formation sites are commonly associated with molecular material belonging to the parental cloud out of which the young stars form. As the young stars evolve, they feed from the

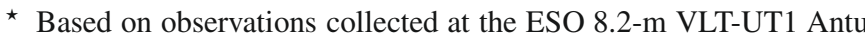
telescope (programme 66.C-0015A). available gas and dissipate it. Embedded clusters emerge from molecular clouds owing to the disruptive action of newly born stars (Matzner \& McKee 2000). Jets and outflows carve cavities into the surrounding molecular material. Scattering off the walls of these cavities allows photons from embedded young stellar objects (YSO) to escape and be detected as near-IR nebulosity, denouncing the presence of one or more YSOs (e.g. Yun et al. 1993; Whitney et al. 1993, 2003).

Furthermore, since most gas and dust that exist in the Galaxy are confined to the Galactic disc, and are most abundant toward the inner Galaxy (Clemens et al. 1988), it is of particular interest to search for and characterise the star formation ocurring near the "edges" of the molecular disc, either along the Galacic disc in the far outer Galaxy or at large vertical distances from the Galactic disc.

As part of our study of young embedded clusters in the third Galactic quadrant (e.g. Yun et al. 2007, 2009), we report here an investigation of a relatively unstudied star formation region (SFR), seen towards the IRAS source IRAS 07383-3325. The position of the IRAS source coincides with that of an optical reflection nebula. The nebula is evident in the Digitized Sky Survey images (especially in that corresponding to the blue O-print) and was discovered by Lauberts (1982), who named it "ESO 368-8" and at that time classified it as an emission nebula. It is also listed in the catalogues of HII regions and reflection nebulae of Brand et al. (1986) and of Magakian (2003) 
(and identified under the names "BRAN 61", "Magakian 287", and "GN 07.38.4").

Optical examination of the Palomar and ESO plates (Brand et al. 1986) did not find any evidence of obscuration towards ESO 368-8. Thus, the molecular nature of this SFR was first revealed in the CO survey of Wouterloot \& Brand (1989) in their survey of IRAS sources beyond the solar circle, where IRAS 07383-3325 is listed as a good detection (No. 1039). We refer to this molecular cloud as WB89-1039, first mapped and probed by us using millimetre spectra, and presented here in Sect. 3.2.

A preliminary search for the young stellar content of an SFR can be performed using $2 \mathrm{MASS}$ images in the $K$ s-band. This has been done by Dutra et al. (2003), who indentified a candidate "open cluster (IROC)" (DBSB 15) located about 22" east of the IRAS source with major and minor axes of $1.9^{\prime}$ and $1.7^{\prime}$. Here, using a combination of deep near-infrared ( $J H K_{\mathrm{S}}$-bands) and optical ( $V$-band) images, we characterise the stellar content of this SFR towards the reflection nebula ESO 368-8/IRAS 07383-3325 and molecular cloud WB89 1039. We confirm the young stellar nature of the candidate open cluster of Dutra et al. (2003). We found a group of stars, spatially coincident with DBSB 15 and extending about $1^{\prime}$ to the east of the IRAS source, involved in infrared nebulosity. We thus report the discovery of a near-infrared reflection nebula seen in the near-IR images.

The Galactic coordinates of this SFR are $l=248.01^{\circ}$, $b=-5.46^{\circ}$ which, together with its estimated distance of $5 \mathrm{kpc}$ (see Sect. 3.2), places this region well below the Galactic plane in the region of the Galactic warp (Momany et al. 2006; May et al. 1997). Thus, this study adds to what has been our relatively poor knowledge of star formation occurring in "frontier" environments, in this case, the lower "edge" of the Galactic disc.

Section 2 describes the observations and data reduction. In Sect. 3, we present and analyse the results. Section 4 discusses the star formation scenario in this region. A summary is given in Sect. 5.

\section{Observations and data reduction}

\subsection{Near-infrared observations}

Near-infrared $\left(J, H\right.$ and $\left.K_{\mathrm{S}}\right)$ images were obtained on 2000 November 11 using the ESO Antu (VLT Unit 1) telescope equipped with the short-wavelength arm (Hawaii Rockwell) of the ISAAC instrument. The ISAAC camera (Moorwood et al. 1998 ) contains a $1024 \times 1024$ pixel near-infrared array and was used with a plate scale of 0.147 arcsec/pixel resulting in a field of view of $2.5 \times 2.5 \mathrm{arcmin}^{2}$ on the sky. For each filter, 6 dithered sky positions were observed. A series of 15 images with individual on-source integration time of $4 \mathrm{~s}$ was taken in the $J$-band. Similarly, series of 20 images, each of $3 \mathrm{~s}$ integration time, were obtained in the $H$ and the $K_{\mathrm{S}}$ bands.

The images were reduced using a set of own IRAF scripts to correct for bad pixels, subtract the sky background, and flatfield the images. Dome flats were used to correct for the pixel-topixel variations of the response. The selected images were then aligned, shifted, trimmed, and co-added to produce a final mosaic image for each band $J H K_{\mathrm{S}}$. Correction for bad pixels was made while constructing the final mosaics that cover about $3.8 \times$ $3.1 \operatorname{arcmin}^{2}$ on the sky.

Point sources were extracted using daofind with a detection threshold of $5 \sigma$. The images were inspected to look for false detections that had been included by daofind in the list of detected sources. These sources were eliminated from the source list and a few additional sources added in by hand. Aperture photometry was made with a small aperture (radius $=3$ pix, which is about the measured FWHM of the point spread function) and aperture corrections, found from bright and isolated stars in each image, were used to correct for the flux lost in the wings of the PSF. The error in determining the aperture correction was $<0.05 \mathrm{mag}$ in all cases. A total of 900,860 , and 969 sources were found to have fluxes in $J, H$, and $K_{\mathrm{S}}$, respectively, and errors $<0.15 \mathrm{mag}$.

The $J H K_{\mathrm{S}}$ zeropoints were determined using faint infrared standard stars (S427-D and S121-E of the LCO/Palomar NICMOS Table of Photometric Standards; see Persson et al. 1998) and checked with those obtained using 2MASS stars brighter than $K_{\mathrm{S}}=13.5$ mag from the 2MASS All-Sky Release Point Source Catalogue (Skrutskie et al. 2006; Cutri et al. 2003). Photometry errors range from about 0.08 mag for the bright stars to $0.15 \mathrm{mag}$ for the fainter ones. From the histograms of the $J H K_{\mathrm{S}}$ magnitudes, we estimate the completeness limit of the observations to be roughly $20.0 \mathrm{mag}$ in the $J$-band, and $18.5 \mathrm{mag}$ in the $H$ and $K_{\mathrm{S}}$ bands.

\section{2. $\mathrm{CO} \mathrm{mm}$ line observations}

Millimetre single-dish observations of IRAS 07383-3325 were carried out at the $15 \mathrm{~m}$ SEST telescope (ESO, La Silla, Chile) during three observational campaigns in 2001 May, 2002 April, and 2002 December. Starting from the $(0,0)$ reference position corresponding to the celestial coordinates of the IRAS source, maps were obtained in the rotational lines of ${ }^{12} \mathrm{CO}(1-0)$ (115.271 GHz), ${ }^{13} \mathrm{CO}(1-0)(110.201 \mathrm{GHz})$, and ${ }^{13} \mathrm{CO}(2-1)$ (220.399 GHz), $\mathrm{C}^{18} \mathrm{O}(1-0)(109.782 \mathrm{GHz})$, and $\mathrm{C}^{18} \mathrm{O}(2-1)$ $(219.560 \mathrm{GHz})$. The SEST half-power beam width (HPBW) was $46^{\prime \prime}$ at $110 \mathrm{GHz}$ and $22^{\prime \prime}$ at $230 \mathrm{GHz}$, so that, since the adopted grid spacing was $46^{\prime \prime}$, the ${ }^{13} \mathrm{CO}(2-1)$ and the $\mathrm{C}^{18} \mathrm{O}(2-1)$ line emissions were strongly undersampled. The ${ }^{12} \mathrm{CO}(1-0)$ and ${ }^{13} \mathrm{CO}(2-1)$ maps are composed of $8 \times 9$ pointings, while the ${ }^{13} \mathrm{CO}(1-0), \mathrm{C}^{18} \mathrm{O}(1-0)$, and $\mathrm{C}^{18} \mathrm{O}(2-1)$ maps are smaller $(5 \times 5,6 \times 5$, and $3 \times 3$ pointings, respectively $)$.

The adopted integration times were between $t_{\text {int }}=60 \mathrm{~s}$ and $t_{\text {int }}=240 \mathrm{~s}$ for each pointing. A high-resolution 2000 channel acousto-optical spectrometer was used as a back end, with a total bandwidth of $86 \mathrm{MHz}$ and a channel width of $43 \mathrm{kHz}$, and was split into two halves to measure both the 115 and $230 \mathrm{GHz}$ receivers simultaneously. At these frequencies, the channel width corresponds to approximately $0.11 \mathrm{~km} \mathrm{~s}^{-1}$ and $0.055 \mathrm{~km} \mathrm{~s}^{-1}$. The spectra were taken in frequency switching mode, recommended to save observational time when mapping extended sources. The antenna temperature was calibrated with the standard chopper wheel method. Pointing was checked regularly towards known circumstellar $\mathrm{SiO}$ masers, and pointing accuracy was estimated to be better than $5^{\prime \prime}$.

The data reduction pipeline was composed of the following steps: $i)$ folding the frequency-switched spectrum; ii) fitting the baseline by a polynomial and subtracting it; iii) co-adding repeated spectra obtained at the same sky position, weighing with the integration time and the inverse of the system temperature; iv) obtaining the main-beam temperature $T_{\mathrm{MB}}$ by dividing the antenna temperature $T_{\mathrm{A}}$ by the $\eta_{\mathrm{MB}}$ factor, equal to 0.7 for the lines at $110-115 \mathrm{GHz}$ and 0.5 for the other lines (at $220 \mathrm{GHz}$ ).

The spectrum baseline rms noise (in $T_{\mathrm{MB}}$ ), averaged over all map positions, has been found to be $0.13 \mathrm{~K}$ for ${ }^{12} \mathrm{CO}(1-0)$, $0.09 \mathrm{~K}$ for ${ }^{13} \mathrm{CO}(1-0), 0.15 \mathrm{~K}$ for ${ }^{13} \mathrm{CO}(2-1), 0.04 \mathrm{~K}$ for $\mathrm{C}^{18} \mathrm{O}(1-0)$, and $0.11 \mathrm{~K}$ for $\mathrm{C}^{18} \mathrm{O}(2-1)$. 


\subsection{Optical V-band observations}

Our optical $V$-band observations were conducted during 1999 December using the $1 \mathrm{~m} \mathrm{YALO}^{1}$ telescope, at the Cerro Tololo Inter-American Observatory in Chile, equipped with the ANDICAM camera. The ANDICAM CCD camera contained $2048 \times 2048$ pixels, but read-out problems meant that only about $1400 \times 1024$ pixels were usable. On a plate scale of $0.3 \mathrm{arcsec} / \mathrm{pixel}$, it provided a field of view of $7.0 \times 5.1 \mathrm{arcmin}^{2}$ on the sky. Standard procedures for CCD image reduction were applied. Source extraction and aperture photometry were performed using IRAF packages. Transformation to the standard system was made using a fit to stars common to our images and to the US Naval Observatory Catalog. The results were checked using the spectrophotometric data of Fitzgerald et al. (1976). Their stars Nos. 27 and 28 fall in our $V$-band image and their magnitudes are compatible (within $0.1 \mathrm{mag}$ ) with the results of our photometry.

\section{Results and discussion}

\subsection{Optical nebula and stars}

Figure 1 shows the central region of the $V$-band image towards ESO 368-8/IRAS 07383-3325. At optical wavelengths, this region of the sky does not look particularly spectacular which justifies the relatively little attention that has been devoted to it. At the centre of the image, a group of a few stars is seen towards optical nebulosity. Basically, this optical nebula appears to clearly involve 3 stars with similar magnitudes and possibly one or two fainter stars. This can be more clearly seen in Fig. 2 where the 3 stars are labelled A-C. The position of the IRAS source lies among this group of optical stars (but does not coincide with any) and well within the optical nebula. We name these stars here as "the optical nebula stars". On the other hand, the IRAS ellipse error (not drawn in the figure) encompasses more than one star. Given the large IRAS beams, it is likely that all of these optical nebula stars contribute to the IRAS fluxes.

We note that, in our images, the optical nebula ESO 368-8 extends for about $1^{\prime}$ diametre (extension measured down to a level of $8 \sigma$ above the background sky brightness; down to $3 \sigma$, the size increases just slightly, to $70^{\prime \prime}$ ), much smaller than the quoted size of $4^{\prime}$ by Brand et al. (1986).

Table 1 gives our photometry of the three brightest optical nebula stars in the optical $V$-band and in the near-infrared $J H K_{\mathrm{S}}$-bands.

\subsection{Molecular gas and distance}

$\mathrm{CO}$ emission has been detected toward this region confirming the presence of a molecular cloud. In Fig. 3, we present the $\mathrm{CO}(J=1-0),{ }^{13} \mathrm{CO}(J=1-0)$, and $\mathrm{C}^{18} \mathrm{O}(J=1-0)$ spectra obtained towards the position of the map where the $\mathrm{CO}$ emission is strongest: the $(+46,0)$ position of the map, or about $46^{\prime \prime}$ east of IRAS 07383-3325. CO lines are detected with LSR velocities in the range of $V_{\mathrm{LSR}} \simeq 47-57 \mathrm{~km} \mathrm{~s}^{-1}$. The ${ }^{12} \mathrm{CO}(1-0)$ spectrum peaks at $V_{\mathrm{LSR}}=51.3 \mathrm{~km} \mathrm{~s}^{-1}$, while those of ${ }^{13} \mathrm{CO}$, and $\mathrm{C}^{18} \mathrm{O}$ lines peak at 52.1 and $51.6 \mathrm{~km} \mathrm{~s}^{-1}$, respectively; these values represent the velocities of the gas component in this molecular cloud.

The kinematic heliocentric distance of this cloud can be derived from the peak radial velocities of the spectra. This

${ }^{1}$ YALO is the Yale-AURA-Lisbon-Ohio consortium (Bailyn et al. 1999).

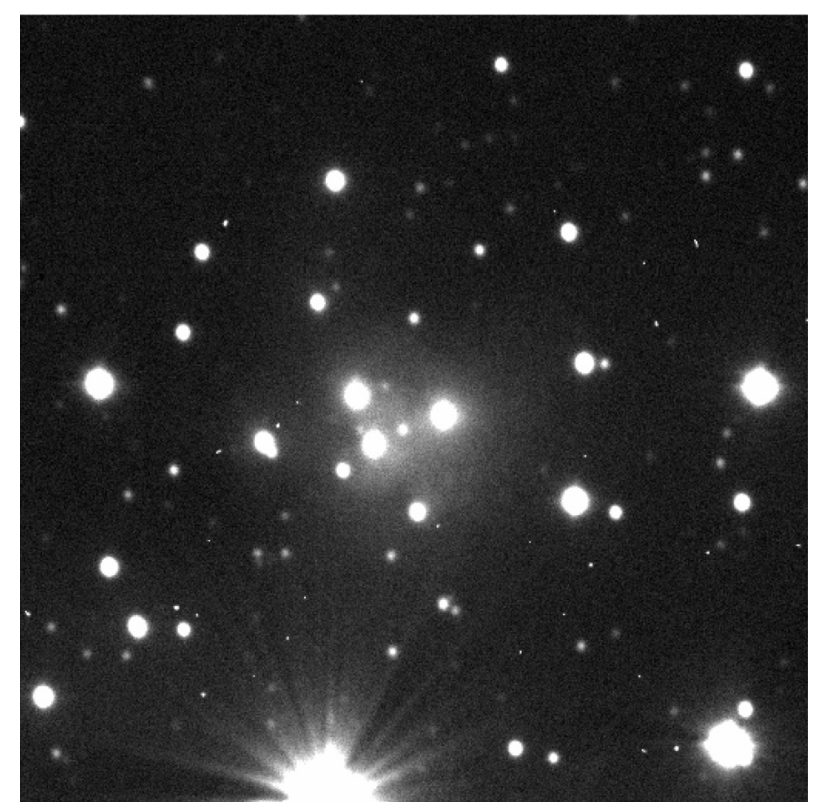

Fig. 1. Central region of the $V$-band image towards ESO 3688/IRAS 07383-3325 (corresponding approximately to the region of the cluster DBSB 15, proposed by Dutra et al. (2003)). Notice the central group of stars involved in optical nebulosity. This region covers about $2.56 \times 2.56 \mathrm{arcmin}^{2}$ and is centred on the IRAS source. North is up and east to the left.

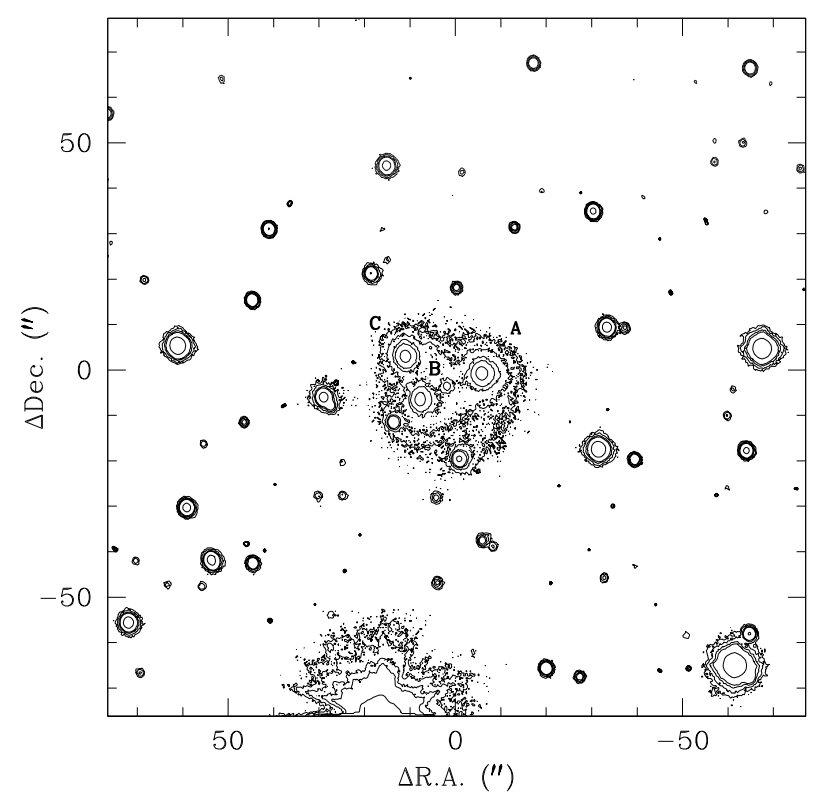

Fig. 2. Isophotes of the $V$-band image towards ESO 368-8/IRAS 073833325 , with labels $(\mathrm{A}-\mathrm{C})$ referring to the 3 brightest optical nebula stars (see text). The contour levels are 8,12, 20,30, and 200 $\sigma$ above the background sky value. The IRAS source is at the $(0,0)$ position.

can be done applying the circular rotation model by Brand \& Blitz (1993). Using $V_{\mathrm{LSR}}=51.3 \mathrm{~km}^{-1}$, a distance $d \simeq$ $5.0 \mathrm{kpc}$ is obtained. Given the Galactic longitude, this implies a Galactocentric distance of $11.4 \mathrm{kpc}$ to the cloud, in the outer Galaxy. We estimate a distance uncertainty of up to $20 \%$ due to uncertainties in the rotation curve and possible streaming motions.

Given the Galactic latitude, the numbers above imply a vertical distance to the Galactic plane (defined by $b=0^{\circ}$ ) of about $500 \mathrm{pc}$, well below it and within the region of the Galactic 
Table 1. Photometry of the optical nebula stars.

\begin{tabular}{lccccccc}
\hline \hline ID & Multiplicity & RA & $\begin{array}{c}\text { Dec } \\
(2000)\end{array}$ & $m_{V}$ & $m_{K_{\mathrm{S}}}$ & $\left(H-K_{\mathrm{S}}\right)$ & $\left(J-K_{\mathrm{S}}\right)$ \\
\hline $\mathrm{A}$ & $\ldots$ & 074014.7 & -333234 & 14.90 & 12.46 & 0.24 & 0.65 \\
$\mathrm{~B}^{a}$ & 1 & 074015.8 & -333239 & 15.27 & 13.04 & 0.29 & 0.95 \\
& 2 & 074015.8 & -333240 & 15.27 & 12.36 & 0.26 & 0.76 \\
$\mathrm{C}$ & $\ldots$ & 074016.1 & -333231 & 15.20 & 13.66 & 0.16 & 0.45 \\
\hline
\end{tabular}

Notes. ${ }^{(a)}$ This star is double, as revealed by our near-IR images. The $V$-band magnitude quoted here corresponds to the combined flux of both stars, unresolved in the optical image.

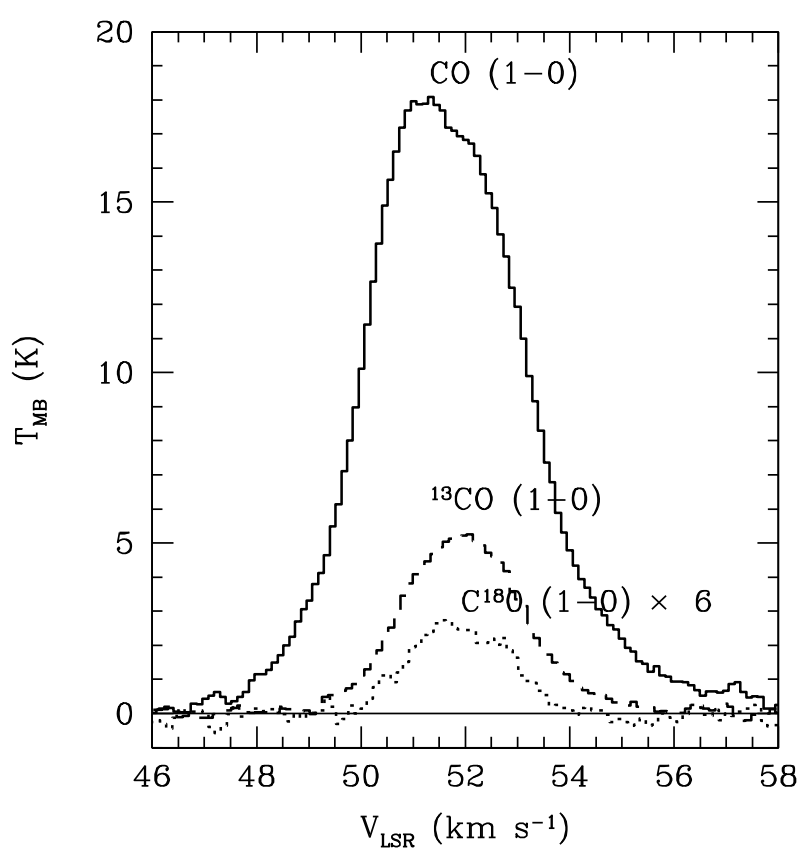

Fig. 3. $J=1-0$ spectra of $\mathrm{CO}$ isotopes towards the position of the map where the $\mathrm{CO}$ emission is strongest (about 46" east of IRAS $07383-3325)$. The ${ }^{12} \mathrm{CO}(1-0)$ line peaks at an LSR velocity of about $51.3 \mathrm{~km} \mathrm{~s}^{-1}$. The $\mathrm{C}^{18} \mathrm{O}(1-0)$ line has been multiplied by a factor 6 .

warp (May et al. 1997). The spatial location of this molecular cloud, both at a relatively large heliocentric distance in the outer Galaxy and far below the Galactic plane, suggests that there are not many background stars (behind the cloud), as discussed in Sect. 3.3.2.

\subsubsection{Molecular gas distribution: a new dense cloud core}

The contour maps of the ${ }^{12} \mathrm{CO}(1-0)$ and ${ }^{13} \mathrm{CO}(1-0)$ integrated intensities $I=\int T_{\mathrm{MB}} \mathrm{d} v$ are shown in Fig. 4. The CO emission is resolved and confined to the mapped region. Our CO maps presented here represent the totality of the WB89-1039 cloud, observed with unprecedented resolution. Only lines characterized by a signal-to-noise ratio $>3$ were considered as genuine emission. The cloud appears slightly elongated approximately in the direction east-west. In the eastern part, there is a peak of strong emission in a core-like or nucleus region; in the western part of the cloud, the emission is narrower and more diffuse in a tail-like fashion. Thus, we identify a new dense cloud core that we designate by "PY10 dense core".

In all maps, the $\mathrm{CO}$ emission peaks at the $(+46,0)$ (about 46" east of IRAS 07383-3325), and decreases steeply in all directions. The shape of the strong $\mathrm{CO}$ emission is thus

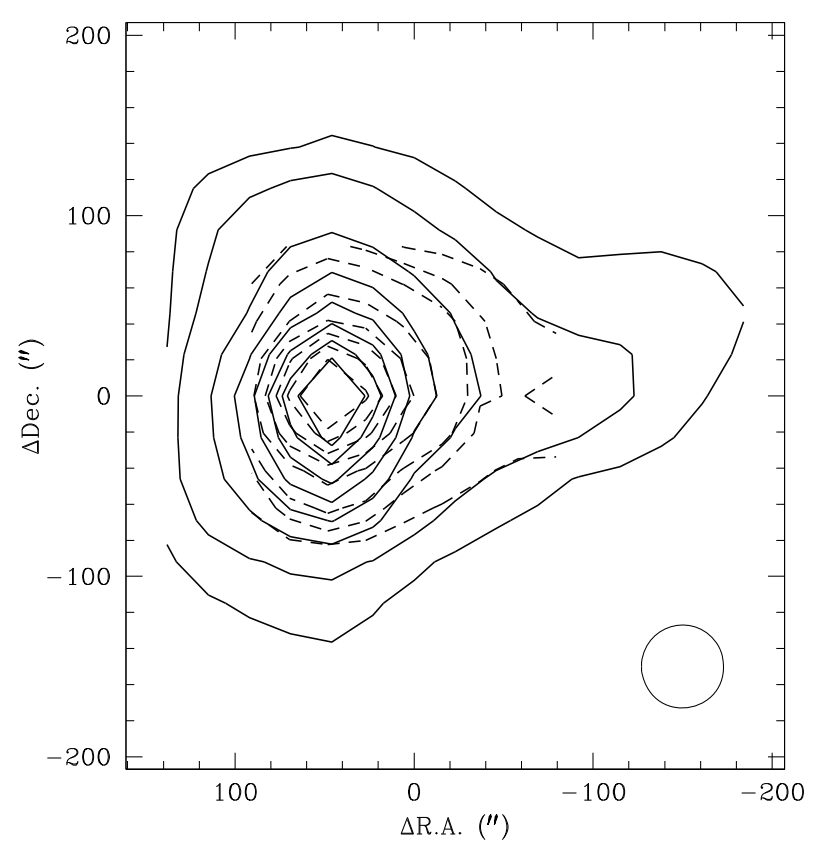

Fig. 4. Smoothed contours of ${ }^{12} \mathrm{CO}(1-0)$ (solid line) and of ${ }^{13} \mathrm{CO}(1-0)$ (dashed line) integrated intensities, in the velocity range between 47 and $57 \mathrm{~km} \mathrm{~s}^{-1}$, observed in the WB89-1039 and IRAS 07383-3325 region, containing a dense core (PY10) about $46^{\prime \prime}$ east of the IRAS $(0,0)$ position. The two ${ }^{12} \mathrm{CO}(1-0)$ lowest contour levels are at $1 \mathrm{~K} \mathrm{~km} \mathrm{~s}^{-1}(5 \sigma)$ and $3 \mathrm{~K} \mathrm{~km} \mathrm{~s}^{-1}$. Subsequent contours are in steps of $5 \mathrm{~K} \mathrm{~km} \mathrm{~s}^{-1}$. The three ${ }^{13} \mathrm{CO}(1-0)$ lowest contour levels are at $0.3 \mathrm{~K} \mathrm{~km} \mathrm{~s}^{-1}(2.5 \sigma)$ and $0.6 \mathrm{~K} \mathrm{~km} \mathrm{~s}^{-1}$ and $1 \mathrm{~K} \mathrm{~km} \mathrm{~s}^{-1}$. Subsequent contours are in steps of $1 \mathrm{~K} \mathrm{~km} \mathrm{~s}^{-1}$. The SEST beam at $115 \mathrm{GHz}$ is displayed in the lower right corner. The IRAS source is at the $(0,0)$ position.

approximately circular and centred on the $(+46,0)$ position. We name this position the "WB89-1039 cloud centre", or the "PY10 core centre". This cloud position is where the gas is warmest and has the highest column density. The maps of $\mathrm{C}^{18} \mathrm{O}(1-0)$ and $\mathrm{C}^{18} \mathrm{O}(2-1)$ are not shown because detection of these lines is practically unresolved, restricted to one map position only, the PY10 centre position.

Figure 5 shows the contour map of the ${ }^{12} \mathrm{CO}(1-0)$ line widths. The line widths across the cloud vary from $1.0 \mathrm{~km} \mathrm{~s}^{-1}$, at the positions where the gas is more quiescent with narrow lines well fitted by Gaussians, to $3.4 \mathrm{~km} \mathrm{~s}^{-1}$ at the PY10 core centre where a peak is located. At the $(0,0)$ IRAS position, the line width is $1.8 \mathrm{~km} \mathrm{~s}^{-1}$, close or intermediate to the value of the quiescent gas. Thus, interestingly, the line widths at the PY10 core centre experience a large increase when compared with neighbouring positions, implying unusual dynamical activity, possibly with energetic embedded sources stirring up the gas. 


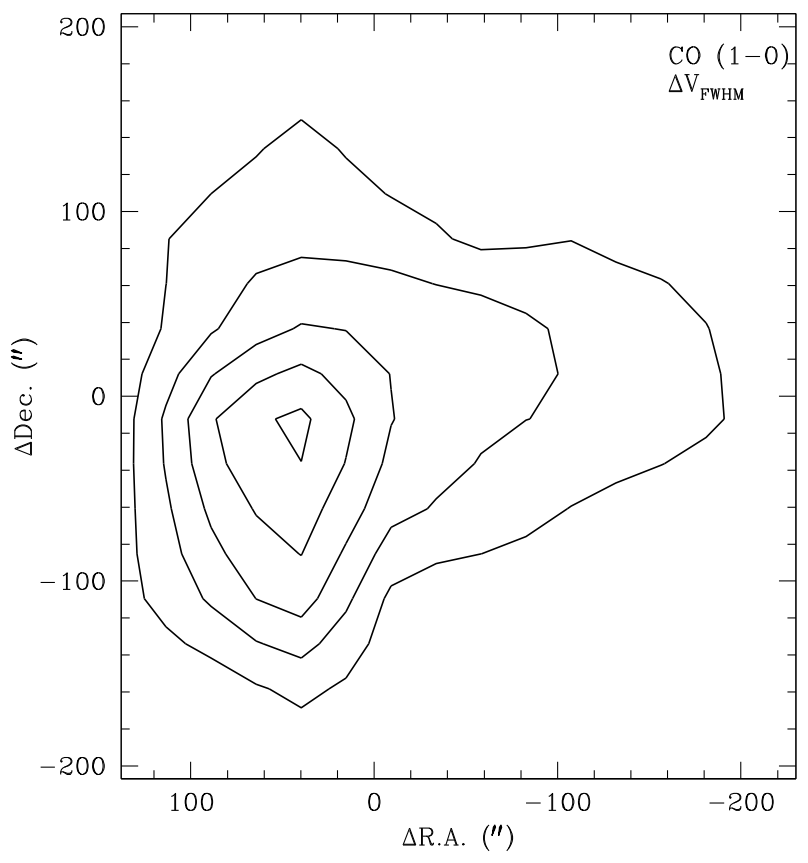

Fig. 5. Smoothed contours of ${ }^{12} \mathrm{CO}(1-0)$ line widths observed in the same region as Fig. 4. The lowest contour is at $0.6 \mathrm{~km} \mathrm{~s}^{-1}$. Subsequent contours are in steps of $0.6 \mathrm{~km} \mathrm{~s}^{-1}$. The IRAS source is at the $(0,0)$ position. The PY10 dense core centre, at the $(+46,0)$ position, is also where the line width peaks.

Taken together, these results suggest that the star formation taking place in this small molecular cloud has lead to the formation of a group of young stars, the optical nebula stars, at the location of the IRAS source. As a result, the molecular gas at this position has been spent and dissipated. However, to the east there is much denser molecular gas, peaking at the PY10 core centre, about $46^{\prime \prime}$ east of the IRAS source, and showing signs of dynamical activity and stirring, possibly caused by additional optical invisible sources, thus indicating current star formation activity, as demonstrated below.

\subsubsection{Molecular cloud mass and optical extinction}

A few different methods can be applied to estimate the cloud mass using line emission of molecular tracers, in this case the $\mathrm{CO}$ isotopes. A first estimate can be made using the empirical correlation between the ${ }^{12} \mathrm{CO}(1-0)$ integrated intensity and the $\mathrm{H}_{2}$ column density along the line of sight, $N_{i, j}$, at position $(i, j)$ :

$N_{i, j}\left(\mathrm{H}_{2}\right)=X \int T_{\mathrm{MB}} \mathrm{d} v \quad\left(\mathrm{~cm}^{-2}\right)$,

where the constant $X$ has been determined empirically. We use the value of $1.9 \times 10^{20} \mathrm{~cm}^{-2}\left(\mathrm{~K} \mathrm{~km} \mathrm{~s}^{-1}\right)^{-1}$ derived by Strong \& Mattox (1996). To obtain the mass from the column density, we also used the "typical" value of 2.72 (Allen 1973) for the mean molecular mass $\mu$. We obtain a mass of $M_{\mathrm{CO}}=1.6 \times 10^{3} M_{\odot}$ for the mapped area.

Furthermore, the derived $N\left(\mathrm{H}_{2}\right)$ value of the column density towards any map position can be used to calculate the optical extinction $A_{V}$ (see Bohlin et al. 1978) towards that position. For the $(0,0)=$ ESO 368-8/IRAS position and for the $(+46,0)=$ PY10 core centre position, using the relation $N\left(\mathrm{H}_{2}\right) / A_{V}=9.4 \times$ $10^{20} \mathrm{~cm}^{-2} \mathrm{mag}^{-1}$ (Frerking et al. 1982), we obtain $A_{V}(0,0)=$ $4.5 \mathrm{mag}$ and $A_{V}(+46,0)=15 \mathrm{mag}$, respectively.
Another method commonly used to estimate the cloud mass consists of assuming LTE conditions and using both an optically thick line and an optically thin line, in this case ${ }^{12} \mathrm{CO}(1-0)$ and ${ }^{13} \mathrm{CO}(1-0)$, respectively. In this method (see e.g. Pineda et al. 2008, for details), the peak main beam temperature of the ${ }^{12} \mathrm{CO}(1-0)$ line is used to derive its excitation temperature, which, for example, is found to be $12.5 \mathrm{~K}$ at the map reference position. Then, assuming that excitation temperatures are the same for both lines, the optical depth and the column density of ${ }^{13} \mathrm{CO}$ are calculated for each line of sight. Finally, to calculate the total mass, it is necessary to adopt $\mathrm{a} \mathrm{H}_{2} /{ }^{13} \mathrm{CO}$ abundance ratios. The "typical" value is $5 \times 10^{5}$ quoted by Dickman (1978).

The obtained mass amounts to $M_{{ }^{13} \mathrm{CO}(1-0)}=6 \times 10^{2} M_{\odot}$, which is compatible with what was obtained by means of the previous empirical method. The remaining discrepancy can be explained taking into account that the number of ${ }^{13} \mathrm{CO}(1-0)$ spectra, contributing to the total map mass estimate, is markedly smaller than in the case of ${ }^{12} \mathrm{CO}(1-0)$, due to smaller spatial coverage and lower $S / N$, and considering that the LTE approximation method seems to typically underestimate the ${ }^{13} \mathrm{CO}$ true column densities (Padoan et al. 2000). Finally, we should note that these two lines, ${ }^{12} \mathrm{CO}(1-0)$ and ${ }^{13} \mathrm{CO}(1-0)$, map different parts of the cloud because of their different optical depths, and also that, evidently, the empirical method based on the ${ }^{12} \mathrm{CO}(1-0)$ emission is quite reliable in a statistical way, but for individual clouds it may give inaccurate results. Therefore, given these considerations, the two mass estimates derived here can be considered in good agreement.

The column density of ${ }^{13} \mathrm{CO}$ can also be used to estimate the optical extinction $A_{V}$ towards the cloud by applying a linear relation:

$A_{V}=c_{1} N\left({ }^{13} \mathrm{CO}\right)+c_{2}$.

Several estimates of the $c_{1}$ and $c_{2}$ parameters can be found in the literature, varying with the observed region and with the calibration technique. Here, we adopt the results of Frerking et al. (1982) for the Taurus region, $c_{1}=7.1 \times 10^{-16} \mathrm{mag} \mathrm{cm}^{-2}$ and $c_{2}=1.0 \mathrm{mag}$. The obtained visual extinction estimates are $A_{V}=$ $3.6 \mathrm{mag}$ for the $(0,0)$ position and $12 \mathrm{mag}$ for the $(+46,0)$ position, similar to the values obtained from the ${ }^{12} \mathrm{CO}(1-0)$ data.

\subsection{The young stellar population}

Figure 6 presents the ISAAC $J H K_{\mathrm{S}}$ colour composite image obtained towards ESO 368-8/IRAS 07383-3325. The three optical nebula stars are seen as relatively blue objects slightly below the centre of the image. However, the most striking aspect of this figure is the red nebula eastward of the centre of the image. This infrared nebula, very bright in the $K_{\mathrm{S}}$-band and invisible at optical wavelengths, has the shape of a fan (or of a flame) and is analysed in more detail in the next Sect. 3.3.1.

In Fig. 7 (top), we show the relative positions of the molecular material (traced by the contour lines of the CO integrated intensity) and of the stellar content of this region as seen in our deep $K$-band image (greyscale). We notice the good spatial coincidence of the IR nebula and the PY10 dense core. The lower panel of Fig. 7 displays the relative positions of all astronomical sources mentioned here.

Figures 6 and 7 also suggest a slight increase in the surface density of stars in the region surrounding the optical nebula stars and the infrared nebula, when compared with the rest of the image. In addition, there is clearly a large concentration of "red" stars in this region (much brighter in the $K_{\mathrm{S}}$-band than in the 


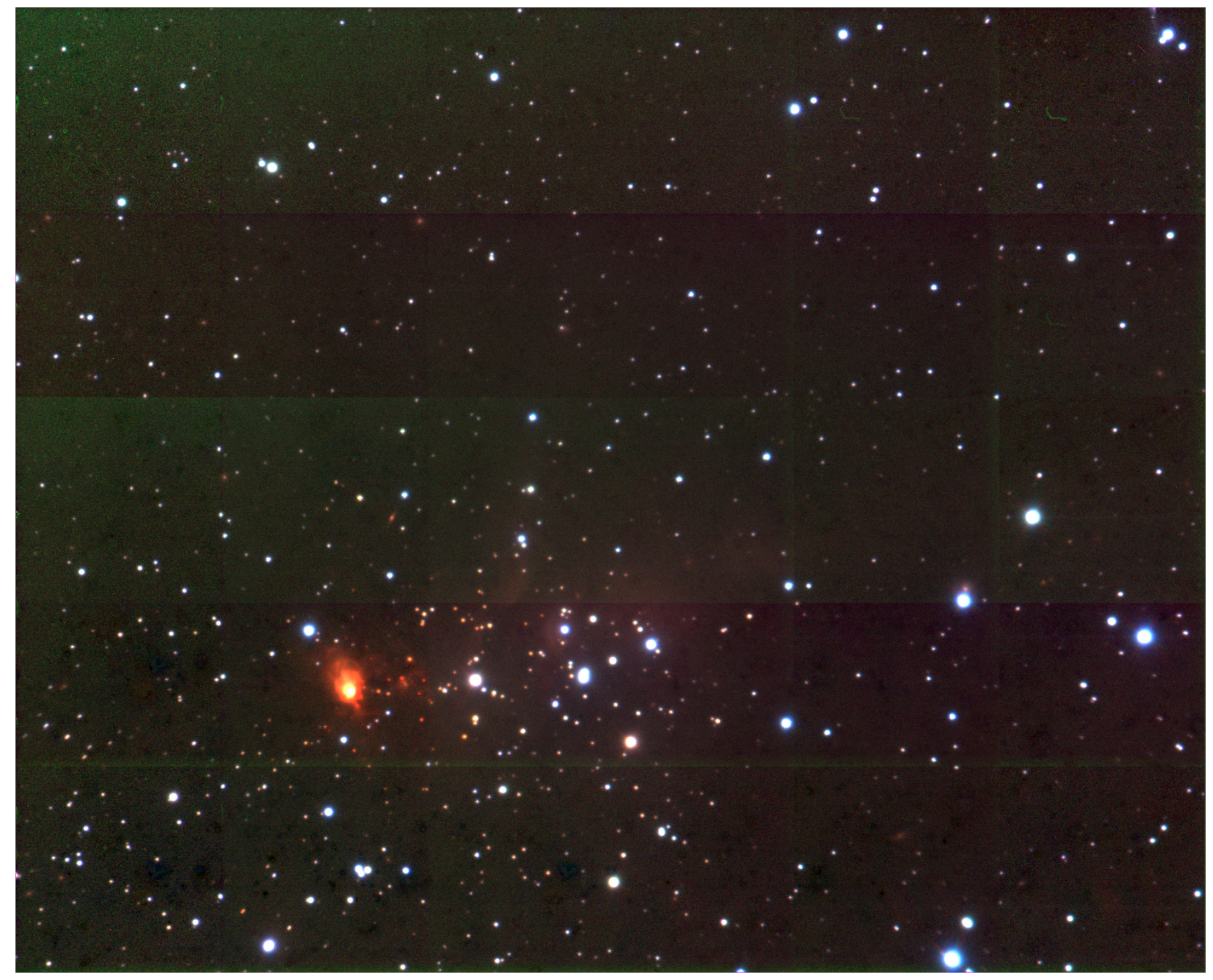

Fig. 6. $J$ (blue), $H$ (green), and $K_{\mathrm{S}}$ (red) colour composite image towards ESO 368-8/IRAS 07383-3325 located in the region of the three optical nebula stars (south of centre). Notice the bright and very red near-infrared nebula (east of centre). The image covers about $3.8 \times 3.1 \mathrm{arcmin}^{2}$. North is up and east to the left.

$J$ or $H$-bands). These aspects will be quantified in Sect. 3.3.1 but, taken together, they suggest a possible small stellar cluster embedded in a region of local enhanced extinction.

\subsubsection{The infrared nebula}

In Fig. 8, we present the isophotes of the $J H K_{\mathrm{S}}$-band images, zoomed-in to show the details of the extended nebular emission seen about $46^{\prime \prime}$ east of the IRAS source. This location coincides with the peak of the molecular emission and of the CO line width map (the cloud centre or PY10 core centre). Thus, we name this IR-nebula "PY10-IRN".

PY10-IRN is very bright and extended in the $K_{\mathrm{S}}$-band image, becoming fainter and shrinking in size as we progress towards the $H$ and the $J$ bluer wavelength images. In each of the nearIR images, the nebula does not contain a detected point-source because the full width at half maximum (FWHM) at any point within the nebula is much greater than the FWHM of the real point sources measured across the images. This indicates that we are not seeing the PY10-IRN nebula illuminator source and that there is no direct line-of-sight to it. Instead, the nebulosities seen in the images are composed of light scattered off the walls of cavities carved in the cloud by jets or outflows and into our line-of-sight.

During the star formation process, the envelope and the molecular cloud core where a young embedded source resides is excavated by a stellar jet and molecular outflow, which are typically most intense during the earliest evolutionary stages of YSOs: Class 0 (Andre et al. 1993), and Class I (Adams et al. 1987). In the Class 0 stage, the source still has to accrete the bulk of its final mass. In the Class I stage, the source is accreting matter from a circumstellar optically thick disc and is also surrounded by an infalling envelope. The presence of these cavities allows radiation from the central embedded object to escape via scattering off the walls of the cavities. Depending on the inclination angle of the cavity axis of symmetry to the line-of-sight, different shapes result for the nebulae that have been found associated to young embedded sources. This can be seen in models of embedded infrared nebulae that have been produced by Lazareff et al. (1990), Whitney et al. (1993), Whitney et al. (2003), and Stark et al. (2006). The shape of our IR-nebula can be compared with these models. It most resembles models Nos. 13 or 14 of 
P. M. Palmeirim and J. L. Yun: Star formation towards ESO 368-8
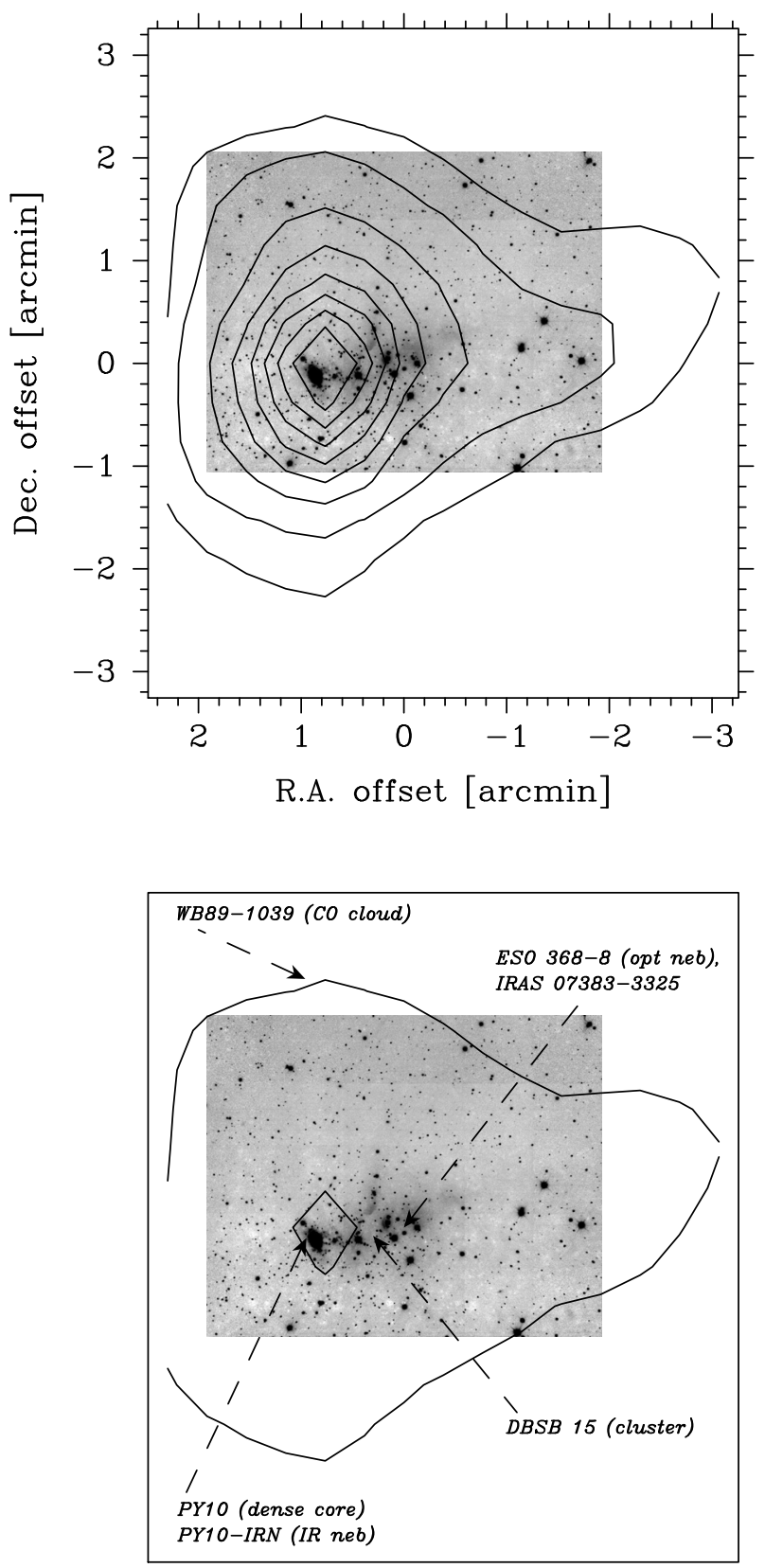

Fig. 7. (Top): smoothed contours of ${ }^{12} \mathrm{CO}(1-0)$ integrated intensity superimposed on the $K_{\mathrm{S}}$-band image. The axes give the coordinates relative to the IRAS source. (Bottom): the spatial position of all the relevant objects: 1) the WB89-1039 CO cloud delineated by the lowest contour of our CO map; 2) the PY10 dense core delineated by the highest CO contour; 3) the DBSB 15 cluster; 4) the ESO 368-8 optical nebula coincident with the IRAS source; and 5) the new PY10-IRN nearinfrared nebula.

Whitney et al. (1993) (their Fig. 4b), which corresponds to an embedded source in a cilindrical cavity with moderate optical depth and an inclination angle (of the polar axis) to the line-ofsight of $\cos i=0.6$. Similarly, the Stark et al. (2006) models suggest that there is a curved or conical outflow cavity viewed at about $60^{\circ}$ inclination.

A simple astrometric analysis of the location of the peak intensity of the nebula in each of the $J H K_{\mathrm{S}}$ images supports the idea that we are seeing scattered light. The positions of the peaks in the $J H K_{\mathrm{S}}$ images are not coincident. Instead they are offset by about $0.12^{\prime \prime}$ and lie close to a straight line, with the values of the
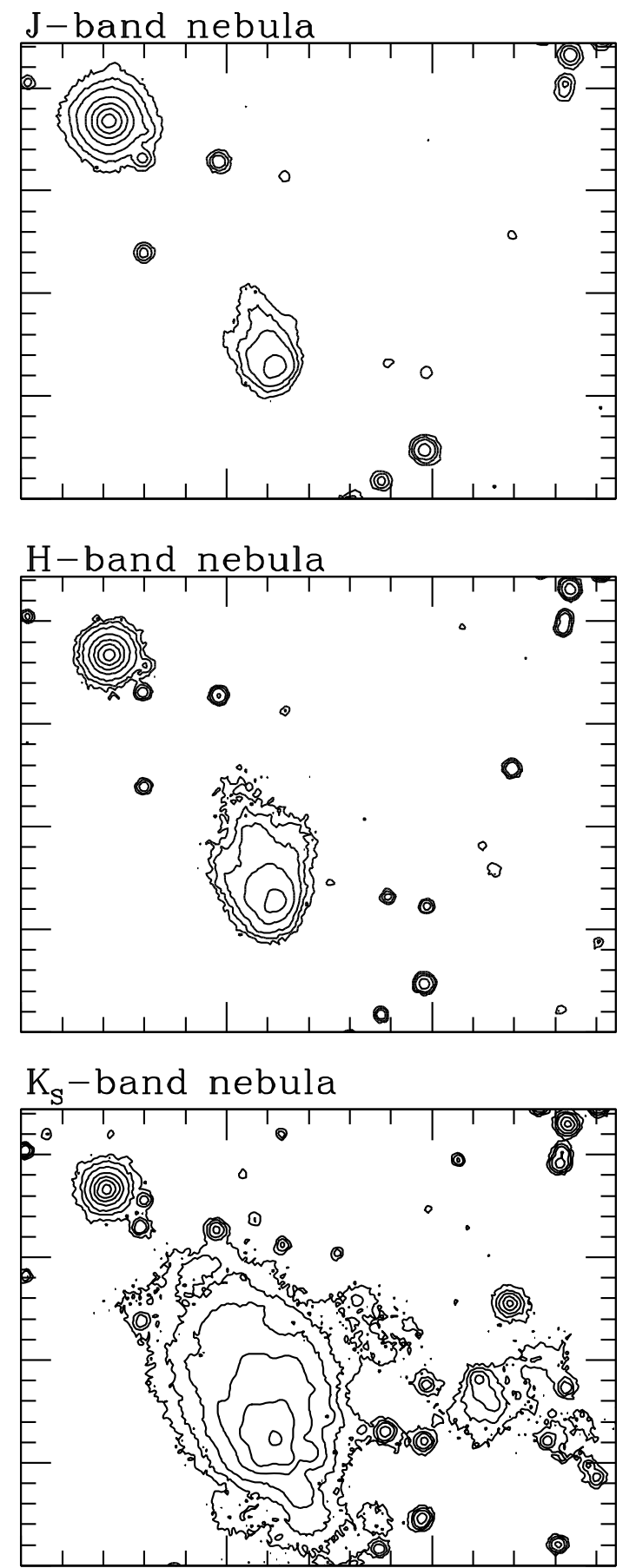

Fig. 8. Isophotes of the $J H K_{\mathrm{S}}$-band images towards a newly discovered near-infrared nebula ("PY10-IRN", coincident with the PY10 core centre, located about 1' east of ESO 368-8/IRAS 07383-3325). The small ticks on the axes indicate the size of $1^{\prime \prime}$. North is up and east to the left. Contour levels are at 5, 10, 20,60, 180, 600, and $2400 \sigma$ above the sky background.

offsets correlating with the wavelength (i.e., the $H$-band peak is offset about $0.12^{\prime \prime}$ from the $K_{\mathrm{S}}$-band peak and the $J$-band peak is offset about $0.12^{\prime \prime}$ from the $H$-band peak approximately in a straight line). The direction of this line is remarkably similar to the direction of the elongation of the $K_{\mathrm{S}}$-band nebula. This behaviour is indicative of scattered light.

In order to provide an estimate of the relative brightnesses of the nebula in the $J H K_{\mathrm{S}}$-bands, we performed aperture photometry of the nebula, for a circular aperture centred on the 


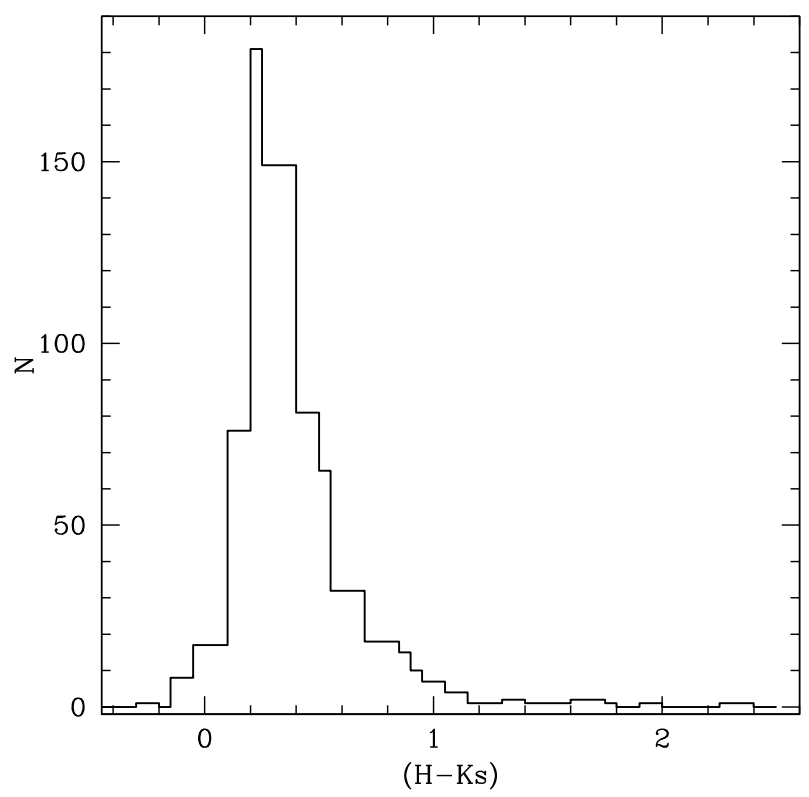

Fig. 9. Histogram of the observed $\left(H-K_{\mathrm{S}}\right)$ colours. The well-defined peak is composed of foreground field sources ("blue" sources with colours $\left.\left(H-K_{\mathrm{S}}\right) \leq 0.8\right)$. The red wing of the distribution is composed of sources spatially concentrated in the region where the molecular cloud is present (see Fig. 10).

location of the peak emission, and an aperture radius equal to the one adopted for the true stars in the images. The results are: $J_{(\mathrm{PY} 10-\mathrm{IRN})}=16.9, H_{(\mathrm{PY} 10-\mathrm{IRN})}=15.1$, and $K_{\mathrm{S}(\mathrm{PY} 10-\mathrm{IRN})}=13.7$. Assuming that the coulours of the nebula are the same as the colours of its illuminator embedded YSO, we obtain $(J-H)=$ $1.8,\left(H-K_{\mathrm{S}}\right)=1.4$. These colours are also compatible with the source being in the Class I stage of low-mass YSOs (Lada \& Adams 1992).

\subsubsection{Reddening and the embedded stellar population}

Figure 9 shows the histogram for the observed $\left(H-K_{\mathrm{S}}\right)$ colours of the sources detected in both the $H$ and the $K_{\mathrm{S}}$-band images. In the range of 0 to about 0.8 , the curve is close to symmetric and most sources here correspond to field main-sequence stars (located in front of the molecular cloud). The spread in $\left(H-K_{\mathrm{S}}\right)$ values, about the peak value of $\left(H-K_{\mathrm{S}}\right)=0.35$, stems from the range of intrinsic colours of main-sequence stars and to low values of variable foreground extinction in the lines of sight of each source. The mean value of the $\left(H-K_{\mathrm{S}}\right)$ colours within this peak (for $\left.\left(H-K_{\mathrm{S}}\right) \leq 0.8\right)$ is 0.33 , with a standard deviation of 0.15 . The spatial location of these peak "blue" sources is seen in Fig. 10 (upper panel) where they are represented by blue open circles. Red filled circles, on the other hand, represent sources in the red tail of the $\left(H-K_{\mathrm{S}}\right)$ histogram, the "red" sources. The blue sources are scattered randomly and uniformly across the image, whereas the red sources are concentrated in the region of the molecular cloud. Taken together, these results strongly indicate that most red sources are objects associated to the cloud, either located behind the cloud or being embedded in the cloud and possibly containing near-infrared excess emission from circumstellar material. We argue here that, due to the location of this molecular cloud well below the Galactic plane and the sparsity of stars in the Galactic halo, few sources seen in the near-infrared images are background sources.
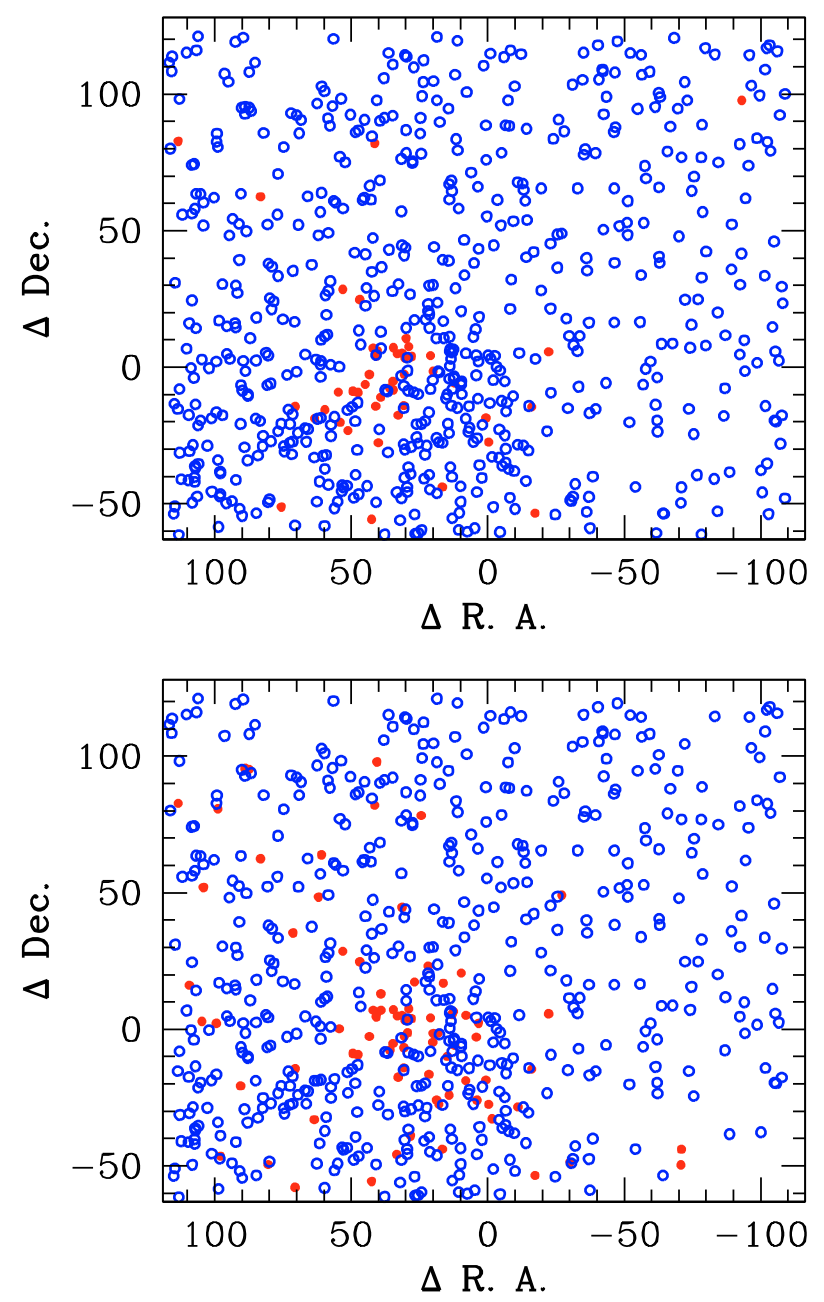

Fig. 10. (Top): spatial distribution of all the sources seen both in the $H$ and in the $K_{\mathrm{S}}$ band images. Blue open circles represent sources with values of $(H-K)$ lower than 0.8 . Red filled circles represent sources with values of $(H-K)$ higher than 0.8 . This plot is centred on the IRAS point source. (Bottom): spatial distribution of all the sources seen simultaneously in the $J, H$ and $K_{\mathrm{S}}$ band images. Red filled circles represent sources to the right of the reddening band (see next figure). Blue open circles represent sources located inside the reddening band.

For the general case, this colour excess of $\left(H-K_{\mathrm{S}}\right)=0.8$ (about equal to the mean value of $\left(H-K_{\mathrm{S}}\right)$ for the field sources plus 3 standard deviations) would just separate the objects into two groups: foreground stars and \{embedded + background stars. However, given that there are very few background stars at this location below the Galactic plane, this reddening effect separates foreground from embedded stars and thus effectively selects YSOs embedded in the cloud. Thus, the large majority of the red sources are likely to be YSOs embedded in the molecular cloud.

Blue sources, on the other hand, may be composed of a mix of foreground field sources and YSOs in a more evolved evolutionary stage. These more evolved young stars, if present, could be pre-main-sequence objects or even intermediate-mass or massive main-sequence stars formed in this cloud, which evolve much faster than their lower-mass siblings formed at the same time. Their higher masses would also contribute to their being bluer and thus not being told apart by red colours. 


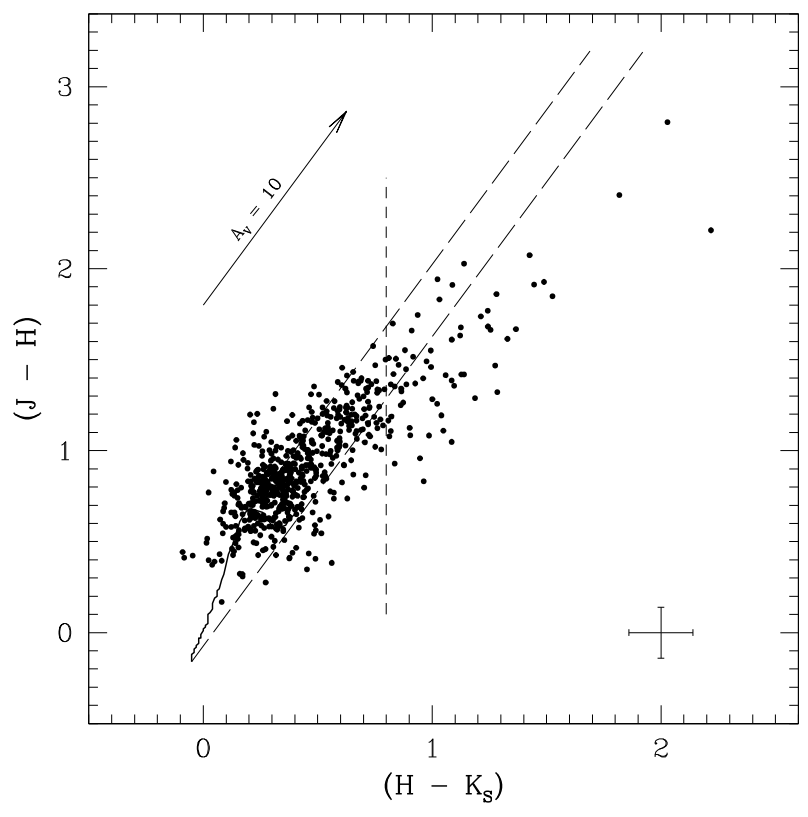

Fig. 11. Near-infrared colour-colour diagram of the region towards IRAS 07383-3325. The solid line represents the loci of unreddened main-sequence stars (Bessel \& Brett 1988), while long-dashed lines indicate the reddening band. The reddening vector indicates the direction of the shift produced by extinction by dust with standard properties. The location of the vertical dashed line, derived from Fig. 9, is at $\left(H-K_{\mathrm{S}}\right)=0.8$. The cross in the lower right corner represents a typical error bar.

\subsubsection{Young stellar objects}

Using the 648 point sources detected in all three $J, H$, and $K_{\mathrm{S}}$-bands, we plotted the near-infrared colour-colour diagram, $(J-H)$ versus $\left(H-K_{\mathrm{S}}\right)$, shown in Fig. 11. Most stars are located within the reddening band where stars appear if they are mainsequence stars reddened according to the interstellar extinction law (Rieke \& Lebofsky 1985), which defines the reddening vector (traced here for $A_{V}=10$ ). Pre-main-sequence YSOs, or massive main-sequence stars recently formed in this region, which have had time to clear the inner regions of their circumstellar discs, lie in this region as well. Giant stars appear slightly above this band. Stars located to the right of the reddening band are likely to be embedded young star objects with infrared excess emission from circumstellar material (Adams et al. 1987).

For the sources that lie inside the reddening band, the highest value of $\left(H-K_{\mathrm{S}}\right)$ is about 1.14 . Using the mean value of $\left(H-K_{\mathrm{S}}\right)=0.33$ for field stars (according to Fig. 9), we obtain a colour excess $E\left(H-K_{\mathrm{S}}\right)=0.81$ due to intra-cloud extinction, which corresponds to about a maximum visual extinction produced by the cloud core of about $A_{V}=13$ (Rieke \& Lebofsky 1985). This value is very similar to those derived towards the densest part of the cloud (position $\left(+46^{\prime \prime}, 0\right)$ or PY10 core) using CO spectra (See Sect. 3.2.2).

The location of the vertical dashed line, derived from Fig. 9, is at $\left(H-K_{\mathrm{S}}\right)=0.80$. The two groups of sources, blue sources with $\left(H-K_{\mathrm{S}}\right)<0.80$ and red sources with $\left(H-K_{\mathrm{S}}\right) \geq 0.80$, are very differently distributed on the colour-colour diagram. A large fraction of the red sources are located outside and to the right of the reddening band, whereas the blue sources mostly occupy the inside of the reddening band. Thus, most red sources are likely to be YSOs. Given their spatial concentration (Fig. 10), these red sources, with $\left(H-K_{\mathrm{S}}\right) \geq 0.80$, seem to represent a small young embedded stellar cluster of about 46 young stars forming in the molecular cloud. The actual number of stars formed in this cloud is likely to be larger for at least three reasons. Firstly, we chose a conservative value of $\left(H-K_{\mathrm{S}}\right)=0.80$ ( $3 \sigma$ above the mean value of the $\left(H-K_{\mathrm{S}}\right)$ of field main-sequence stars). Secondly, the presence of the optical nebula stars seems to indicate that there are some young stars in a more advanced stage of the star formation process, already free of circumstellar material and exhibiting blue colours, thus not pinpointed by our colour selection criterion. Thirdly, there are IR-excess sources with $\left(H-K_{\mathrm{S}}\right)<0.80$ that are YSOs belonging to the cluster. The presence of this YSO population is likewise supported by Fig. 10 (lower panel) where a plot similar to the upper panel is shown. We notice that unlike the upper panel, in the lower panel red filled circles represent sources with IR excess emission (derived from Fig. 11). The distribution of the IR excess stars is similar to the distribution of the large $\left(H-K_{\mathrm{S}}\right)$ sources. Both are concentrated at the location of DBSB 15, between ESO3688/IRAS 07383-3325 and the PY10-IRN nebula (closer to the latter), and trace the young stellar population forming in this cloud core. Thus, as mentioned above, the specific location of this SFR in the Galaxy makes the two approaches illustrated in Figs. 10 almost equivalent in pinpointing young stars.

Given the uncertainty in the number of stars forming in the cloud, we can make a rough estimate of the star formation efficiency of this molecular cloud. Assuming that there are 46 solar mass stars and 3 optical nebula stars that could reach about 6 solar masses each (see next section), we obtain a total stellar mass of $\sim 64 M_{\odot}$. For a total mass of the gas in the cloud between 600 and $1600 M_{\odot}$, we obtain a star formation efficiency in the range of $4-10 \%$. Even though the uncertainty is large, these values are similar to the lower end values of star formation efficiencies found in cluster environments within the local star formation regions (e.g., L1630; Lada 1999).

\section{Discussion}

The two non-spatially coincident nebulae, ESO 368-8 (seen in the optical) and PY10-IRN (seen in the near-IR images), together with a small enhancement of the surface stellar density are the main signs of star formation in the form of a small embedded young cluster in the WB89-1039 molecular cloud. Several aspects make this region particularly interesting. The first aspect is the Galactic location, in the outer Galaxy and well below the Galactic plane. Even though not in the far outer Galaxy (defined as Galactocentric distances $>13.5 \mathrm{kpc}$; this number is based on the radial distribution of $\mathrm{CO}$ emission from the studies of Digel et al. 1996; Heyer et al. 1998), this location shares at least one aspect with the far outer Galaxy: it is close to the "edge" of the Galactic molecular disc (in this case, the lower edge), about $500 \mathrm{pc}$ (vertical distance) below the plane of the Galaxy defined by $b=0^{\circ}$. Interestingly, star formation occurs frequently and with no major differences in these edge environments, both in the far outer Galaxy (e.g. Brand \& Wouterloot 2007; Yasui \& Kobayashi 2008; Yun et al. 2009), and also well below the Galactic plane as shown here. The relatively small number of molecular clouds at this large vertical distance (May et al. 1997) do not appear to significantly affect the occurrence and properties of the star formation process. The only significant difference of these "edge" star formation sites is that they do not seem to form massive stars (e.g. Yun et al. 2007, 2009). In fact, based on the observed fluxes, no massive star is expected to be forming in this region.

In the following, we discuss the nature of the optical nebula stars and the presence of the two non-spatially coincident 


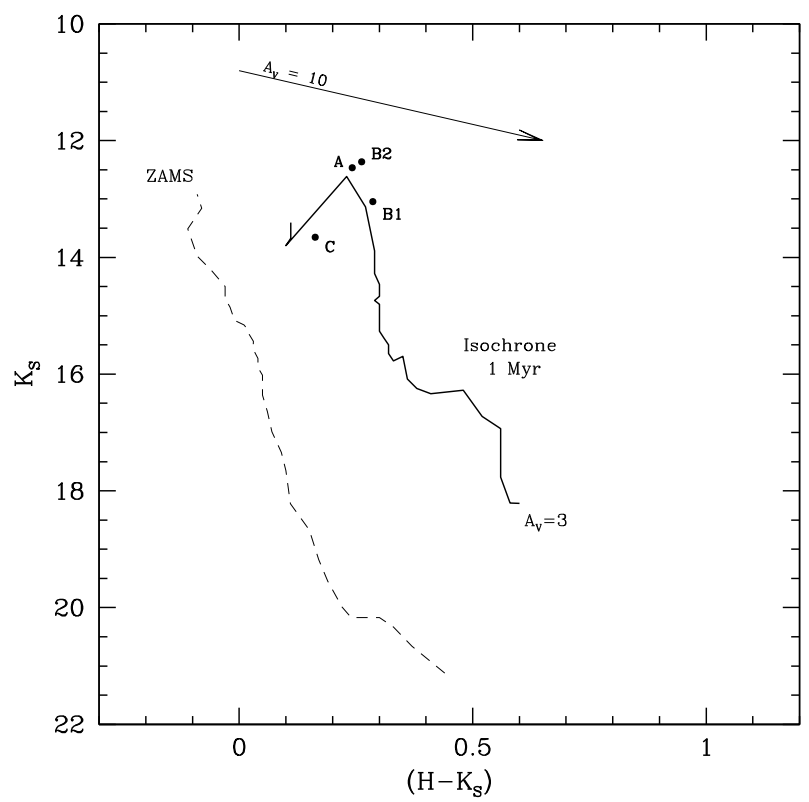

Fig. 12. Colour-magnitude diagram with the positions of the optical nebula stars (A, B1, B2, and C) marked by filled dots. The dashed line is the zero-age main-sequence (ZAMS; Siess et al. 2000) for the cloud distance and with zero extinction. The solid line is the $1 \mathrm{Myr}$ isochrone with extinction $A_{V}=3$. The reddening vector indicates the direction of the shift produced by extinction by dust with standard properties (Rieke \& Lebofsky 1985).

nebulae, focusing especially on what they suggest for the star formation scenario in this molecular cloud.

\subsection{The optical nebula stars}

The nature of the stars involved in the optical nebula can be investigated using the $\left(H-K_{\mathrm{S}}\right), K_{\mathrm{S}}$ colour-magnitude diagram seen in Fig. 12. In this figure, we have marked the positions of the optical nebula stars $(\mathrm{A}-\mathrm{C})$. The optical nebula star B is in fact a double star resolved in our near-IR images (whose stars are named here B1 and B2). The best fit to these sources is achieved using the $1 \mathrm{Myr}$ isochrone of Siess et al. (2000) with extinction $A_{V}=3$. This value is in good agreement with the value of $A_{V}$ derived towards these stars (the $(0,0)$ position) using the CO data (Sect. 3.2.2). Bearing in mind the relatively large uncertainties when fitting isochrones to colour-magnitude diagrams, we estimate that the optical nebula stars have an age close to $1 \mathrm{Myr}$ and are intermediate-mass stars (from spectral type B5 to A9). Furthermore, using $A_{V}=3$, the absolute magnitudes in the $V$-band of the optical nebula stars span values that are a few magnitudes greater than the corresponding values for intermediate-mass main-sequence stars. This is compatible with these stars being pre-main-sequence intermediate-mass stars whose luminosities are still higher than the corresponding ones when they reach the main-sequence.

Figure 13 shows the colour-magnitude diagram for the sources seen in both the $H$ and the $K_{\mathrm{S}}$-bands but excluding those that exhibit signs of excess emission due to circumstellar material (as inferred from Fig. 11). In this way, the location of a star on this diagram depends on its distance (vertical shift), on its extinction (shift along the reddening vector) and on its age (shift given by pre-main-sequence theoretical models of stellar photospheres with no circumstellar emission). The two groups of sources (filled red sources with $\left(H-K_{\mathrm{S}}\right) \geq 0.8$ and open blue

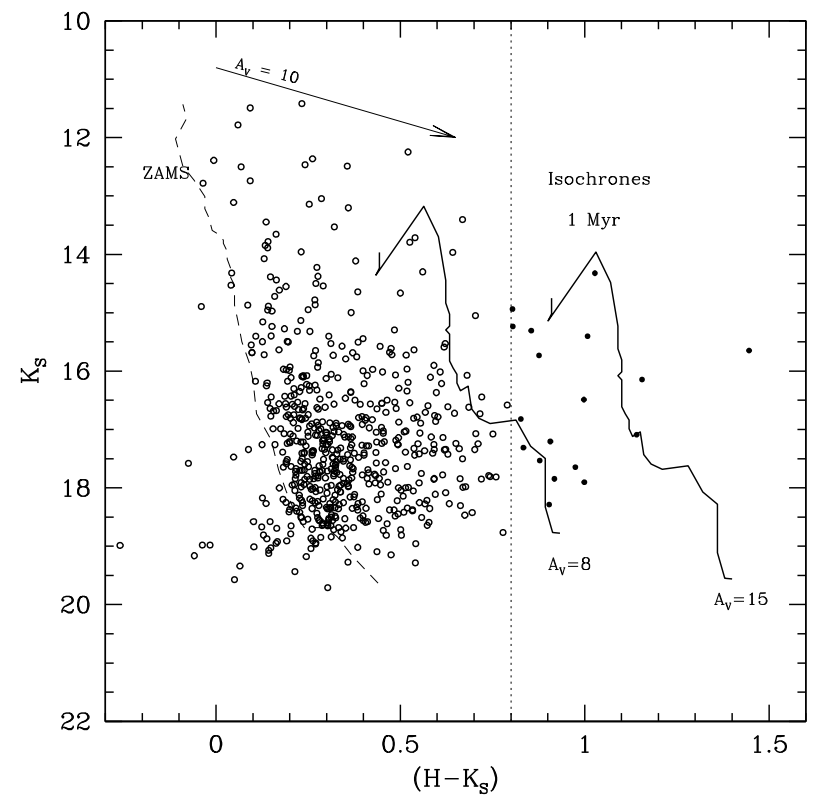

Fig. 13. Colour-magnitude diagram for the sources seen in both the $H$ and the $K_{\mathrm{S}}$-bands with no signs of excess emission due to circumstellar material. The dashed line is the zero-age main-sequence for an arbitrary distance. The two solid lines are the $1 \mathrm{Myr}$ isochrones with extinctions $A_{V}=8$ and $A_{V}=15$, respectively. Filled circles represent the red sources with $\left(H-K_{\mathrm{S}}\right) \geq 0.8$. Open circles represent the blue sources with $\left(H-K_{\mathrm{S}}\right)<0.8$.

sources with $\left.\left(H-K_{\mathrm{S}}\right)<0.8\right)$ are separated by the vertical dotted line. The blue sources represent mostly foreground mainsequence stars, at variable distances and small extinction. The red sources can be best fitted by a $1 \mathrm{Myr}$ isochrone with variable values of extinction, as is known to exist toward embedded sources, between about $A_{V}=8$ and $A_{V}=15$, consistent with the values of $A_{V}$ towards these sources (mostly located around the $(+46,0)$ position) derived from the molecular data (Sect. 3.2.2). Thus, this plot supports the idea that we are in the presence of a young stellar cluster or aggregate with about $1 \mathrm{Myr}$ age and composed of at least some tens of stars.

\subsection{The two reflection nebulae}

The two reflection nebulae, the optical nebula ESO 368-8 and the near-IR nebula PY10-IRN, are separated by about 46" (1.1 pc). Not surprisingly the location of the optical nebula is relatively poor in gas, when compared with the location of the near-IR nebula where the amount of gas (and the extinction) is much greater. Do the two nebulae mark two star formation sites in the same cloud, or can the two nebulae be considered coeval and two manifestations of the same episode of star formation taking place in the cloud? In the latter case, the two different stages of the young stars, the optical nebula stars and the red sources close to the near-IR nebula, could stem from different stellar masses. The optical nebula stars should be sufficiently more massive than the stars forming close to the near-IR nebula. Because of their higher masses, they would have evolved faster and dissipated the cloud material while their lower mass siblings to the east are still heavily embedded and extincted at optical wavelengths.

On the other hand, if the two nebulae represent two noncoeval star formation sites, could this be the case for triggered sequential star formation? Using the gas temperatures from the $\mathrm{CO}$ observations to derive the sound speed in the cloud, we 
conclude that it would take about 2.3 Myr for a pressure wave to travel from the optical nebula to the near-IR nebula (for a projected distance of $1.1 \mathrm{pc}$ between the two nebulae). If the optical nebula stars have triggered star formation at the near-IR location, they had to have done it at least 2.3 Myr ago. This value is of the order or greater than the age derived for the optical nebula stars. This fact, and the ages derived in the previous section, favour the scenario of the nebulae being considered coeval and representing two manifestations of the same single star formation episode. Moreover, the higher velocities inferred from the line widths within the PY10 core, peak at the position of the near-IR nebula. This indicates that the source of gas stirring here is likely to be an embedded source at this position, not a source located far out at the position of the optical nebula where line widths are not enhanced.

\section{Summary}

We present a study of the molecular gas and young stellar population seen towards the region of the reflection nebula ESO 368-8 and the IRAS source IRAS 07383-3325, in the molecular cloud WB89-1039 located in the outer Galaxy and well below the Galactic plane. We confirm the presence of a young stellar cluster (or aggregate of tens of YSOs), identified by Dutra et al. (2003), composed of low and intermediate-mass stars, with no evidence of high-mass stars.

This star formation region is associated with the ESO 368-8 optical reflection nebula and with a near-infrared nebula (PY10-IRN) located about 46" (1.1 pc) from each other. The two nebulae seem to be coeval and to represent two manifestations of the same single star formation episode with about $1 \mathrm{Myr}$ age. The near-IR nebula appears to be composed of scattered light off a cavity carved by previous stellar jets or molecular outflows from an embedded, optically, and near-IR invisible source.

The molecular cloud was fully covered by our $\operatorname{CO}(J=1-0)$ maps and, traced by this line, extends over a region of $\sim 7.8 \times$ $7.8 \mathrm{pc}^{2}$, exhibiting an angular size $\sim 5.4^{\prime} \times 5.4^{\prime}$ and shape (close to circular) similar to spherical (or slightly cometary) globules. Towards the direction of the near-IR nebula, the molecular cloud contains a dense core (PY10) where the molecular gas exhibits the large line widths indicative of a very dynamical state, with stirred gas and supersonic motions that could generate turbulence.

Our estimates of the mass of the molecular gas in this region range from 600 to 1600 solar masses. In addition, the extinction $A_{V}$ towards the positions of ESO 368-8 (optical reflection nebula) and of PY10-IRN (near-IR nebula), derived by means of different column density methods, was found to be $A_{V} \simeq$ 3-4 mag and $A_{V} \simeq 12-15 \mathrm{mag}$, respectively. We estimate a value of $\sim 4-10 \%$ for the star formation efficiency of this molecular region.

Acknowledgements. This work has been partly supported by the Portuguese Fundação para a Ciência e Tecnologia (FCT) and by the European Commission FP6 Marie Curie Research Training Network "CONSTELLATION" (MRTNCT-2006-035890). The research made use of the NASA/IPAC Infrared Science Archive, which is operated by the Jet Propulsion Laboratory, California Institute of Technology, under contract with the National Aeronautics and Space
Administration. This research made use of the SIMBAD database, operated at CDS, Strasbourg, France, as well as SAOImage DS9, developed by the Smithsonian Astrophysical Observatory.

\section{References}

Adams, F. C., Lada, C. J., \& Shu, F. H. 1987, ApJ, 312, 788

André, P., Ward-Thompson, D., \& Barsony, M. 1993, ApJ, 406, 122

Allen, C. W. 1973, Astrophysical Quantities, University of London (London: Athlone Press)

Barnard, E. E. 1913, Lick Obs. Publ., 11

Barnard, E. E. 1927, A Photographic Atlas of Selected Regions of the Milky Way, ed. E. B. Frost, \& M. R. Calvert, Washington, Carnegie Institution of Washington

Bailyn, C., DePoy, D., Agostinho, R., et al. 1999, The First Year of Operations of the YALO Consortium, AAS, 195, 8706

Bessell, M. S., \& Brett, J. M. 1988, PASP, 100, 1143

Bohlin, R. C., Savage, B. D., \& Drake, J. F. 1978, ApJ, 224, 132

Brand, J., \& Blitz, L. 1993, A\&A, 275, 67

Brand, J., \& Wouterloot, J. G. A. 2007, A\&A, 464, 909

Brand, J., Blitz, L., \& Wouterloot, J. G. A. 1986, A\&AS, 65, 537

Bica, E., Dutra, C. M., Soares, J., \& Barbuy, B. 2003, A\&A, 404, 223

Clemens, D. P., Sanders, D. B., \& Scoville, N. Z. 1988, ApJ, 327, 139

Cutri, R. M., Skrutskie, M. F., Van Dyk, S., et al. 2003, Explanatory Supplement to the 2MASS All Sky Data Release, http://www.ipac.caltech.edu/ 2mass/releases/allsky/doc/explsup.html

Digel, S. W., Lyder, D. A., Philbrick, A. J., et al. 1996, ApJ, 458, 561

Dickman, R. L. 1978, ApJS, 37, 407

Dutra, C. M., Bica, E., Soares, J., \& Barbuy, B. 2003, A\&A, 400, 533

Fitzgerald, M. P., Hurkens, R., \& Moffat, A. F. J. 1976, A\&A, 46, 287

Frerking, M. A., Langer, W. D., \& Wilson, R. W. 1982, ApJ, 262, 590

Heyer, M. H., Snell, R. L., Brunt, C., et al. 1998, ApJS, 115, 241

Hubble, E. 1922, ApJ, 56, 400

Lauberts, A. 1982, ESO/Uppsala Survey of ESO(B) atlas, European Southern Observatory (ESO)

Lazareff, B., Pudritz, R. E., \& Morin, J. L. 1990, ApJ, 358, 170

Lada, E. A. 1999, The Origin of Star and Planetary Systems, ed. C. Lada, \& N. Kylafis (Dordrecht: Kluwer), 441

Lada, C. J., \& Adams, F. C. 1992, ApJ, 393, 278

Magakian, T. Y. 2003, A\&A, 399, 141

Matzner, C. D., \& McKee, C. F. 2000, ApJ, 545, 364

May, J., Alvarez, H., \& Bronfman, L. 1997, A\&A, 327, 325

Momany, Y., Zaggia, S., Gilmore, G., et al. 2006, A\&A, 451, 515

Moorwood, A., Cuby, J.-G., Biereichel, P., et al. 1998, Messenger, 94, 7

Padoan, P., Juvela, M., Bally, J., \& Nordlund, A. A. 2000, ApJ, 529, 259

Persson, S. E., Murphy, D. C., Krzeminski, W., Roth, M., \& Rieke, M. J. 1998, AJ, 116, 2475

Pineda, J. E., Caselli, P., \& Goodman, A. A. 2008, ApJ, 679, 481

Rieke, G. H., \& Lebofsky, M. J. 1985, ApJ, 288, 618

Siess, L., Dufour, E., \& Forestini, M. 2000, A\&A, 358, 59

Skrutskie, M., Schneider, S. E., Stiening, R., et al. 1997, in The Impact of Large

Scale Near-IR Sky Surveys, ed. F. Garzon et al. (Dordrecht: Kluwer), 25

Skrutskie, M. F., Cutri, R. M., Stiening, R., et al. 2006, ApJ, 131, 1163

Soares, J. B., \& Bica, E. 2002, A\&A, 388, 172

Soares, J. B., \& Bica, E. 2003, A\&A, 404, 217

Stark, D. P., Whitney, B. A., Stassun, K., \& Wood, K. 2006, ApJ, 649, 913

Strong, A. W., \& Mattox, J. R. 1996, A\&A, 308, L21

Testi, L., Palla, F., \& Natta, A. 1998, A\&AS, 133, 81

van den Bergh, S. 1966, AJ, 71, 990

Whitney, B. A., \& Hartmann, L. 1993, ApJ, 402, 605

Whitney, B. A., Wood, K., Bjorkman, J. E., \& Cohen, M. 2003, ApJ, 598, 1079

Wouterloot, J. G. A., \& Brand, J. 1989, A\&AS, 80, 149

Yasui, C., \& Kobayashi, N. 2008, ApJ, 675, 443

Yun, J. L., Clemens, D. P., McCaughrean, M. J., \& Rieke, M. 1993, ApJ, 408, 101

Yun, J. L., Lopez-Sepulcre, A., \& Torrelles, J. M. 2007, A\&A, 471, 573

Yun, J. L., Elia, D., Palmeirim, P. M., Gomes, J. I., \& Martins, A. M. 2009, A\&A, 500,833 